\title{
FEM Model-Based Investigation of Ultrasonic TOFD for Notch Inspection
}

\author{
Ziqiao Tang*, Maodan Yuan*, Hu Wu*, Jianhai Zhang*, Hak-Joon Kim*, \\ Sung-Jin Song ${ }^{\dagger}$ and Sung-Sik Kang**
}

\begin{abstract}
A two-dimensional numerical model based on the finite element method was built to simulate the wave propagation phenomena that occur during the ultrasonic time of flight diffraction(TOFD) process. First, longitudinal-wave TOFD was simulated, and the numerical results agreed well with the theoretical results. Shear-wave TOFD was also investigated because shear waves have higher intensity and resolution. The shear wave propagation was studied using three models with different boundary conditions, and the tip-diffracted shear-tolongitudinal wave was extracted from the A-scan signal difference between the cracked and non-cracked specimens. This signal showed very good agreement between the geometrical and numerical arrival times. The results of this study not only provide better understanding of the diffraction phenomena in TOFD, but also prove the potential of shear-wave TOFD for practical application.
\end{abstract}

Keywords: Ultrasonic Time of Flight Diffraction, Finite Element Method, Boundary Condition, Wave Propagation

\section{Introduction}

In conventional ultrasonic testing(UT), a piezoelectric transducer as transmitter fires a pulse of narrow ultrasonic beam into the specimen and another transducer as receiver records the signals containing the defect signal and geometry boundary reflection signals. For pulse-echo techniques, it is based on the ideal model that the reflected echo comes from planar features which are suitably angled to give a specular reflection back to the transducer.

The arriving time can be used to locate the defect and its amplitude can be used for defect sizing. However, in practice, it must be quite rare for defects to be exactly normal to the beam and there always exists random oriented defects that will make the wave propagation very complicated. Moreover, the surface of the defect may be rough, which will make the reflection beam produce an angular spread [1]. So amplitude of the reflected pulse may lack some accuracy due to the influence by surface roughness, transparency, and orientation of the defect [2].

When an ultrasonic wave in solid media encounters an obstacle, which is of a few wavelength, some of the wave will be bent into the shadow zone by diffraction. According to Huygens principle, the edge of the obstacle acts like secondary point sources reradiating energy over wider angles. These edge waves can therefore be singled out using suitably positioned ultrasonic transducers. Based on the measurement of the time-of-flight of these diffracted waves at the crack edges, the crack can be accurately sized and located. This is the basic of the time-of-flight diffraction technique $[3,4]$, which has been illustrated in Fig. 1.

Thin-walled structures [5] are often found in aerospace applications such as cryogenic tanks, solid rocket motor casings, robot welding parts, etc and need to be inspected to determine the defect size in order to predict the residual

[Received: December 5, 2013, Revised: January 2, 2014, Accepted: January 7, 2014] *School of Mechanical Engineering, Sungkyunkwan University, Suwon 440-746, Korea **Korea Institute of Nuclear Safety, Daejeon 305-338, Korea †Corresponding Author: sjsong@skku.edu

(c) 2014, Korean Society for Nondestructive Testing 


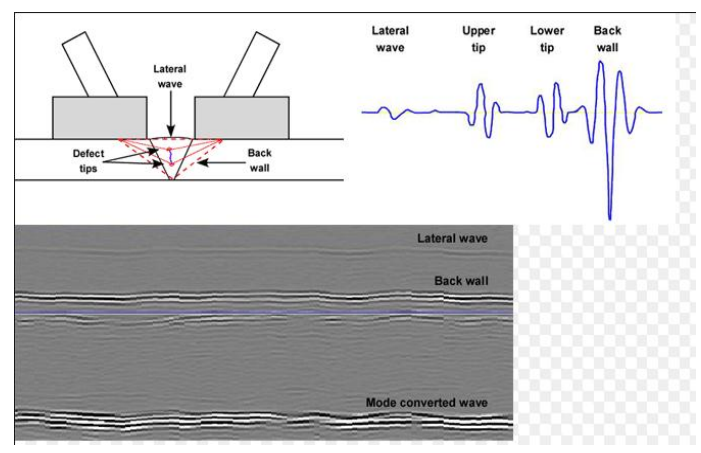

Fig. 1 Principle of conventional TOFD technique and expected A-scan and B-scan signals

lifespan of the components. Some of the difficulties with TOFD inspection of thin sections can be listed as below: 1) whenever ultrasound strikes a surface or defect, some of the energy will be converted to other wave modes (L-S or S-L). This makes difficulty for signal identification; 2) as the thickness of the specimen decreases, the spacing between lateral wave and backwall echo decreases. The crack tip echo (due to L-wave) always lies in the region between lateral and backwall echoes and hence temporal resolution plays a determining role when dealing with thin section. The signals superposition makes TOFD calculations difficult [6]. In our work, thin sections (10 and $13 \mathrm{~mm}$ ) are detected using the TOFD method.

For the above problem, longitudinal and shear wave were selected as the incident beam to model the wave propagation in the material. For longitudinal wave, 1) higher diffraction coefficient than that of shear wave; 2) the speed of longitudinal wave is almost twice of shear wave and so that the primary diffracted signals are longitudinal waves and arrive well ahead of any signals concerned about wave mode conversion. For the shear wave, 1) as the speed is half of P-wave, the space between the lateral wave and backwall reflected wave (S-S) will increase which makes the diffracted signal between them easier to identify; 2) shear wave has a wave length roughly half that of compression waves and therefore offers an enhanced resolution [7].

\section{Background}

When we refer to the complicated ultrasonic wave propagation, numerical method provides a useful way for researching the wave reflection and scattering in solid media [8]. Numerical modeling of wave propagation processes was predominantly the domain of high-level mathematicians experienced in numerical and computational techniques. By changing the input parameters in numerical simulation, we can transfer the complex physical system to the equivalent mathematical form which can simply solved by the computer [9]. Finite element method is effective and convenient in the simulation of some simplified models to investigate the crack inspection. Simulation of ultrasonic waves in a solid media using numerical techniques has been carried out in many studies. Finite element simulation of ultrasonic wave propagation and its interaction with defects have been studied by Ludwig and Lord [10]. Simulation of the TOFD technique using finite element method has been carried out by Baskaran et al [4]. They used the finite element package ANSYS to model the propagation of ultrasonic waves in a thin cracked two dimensional specimen. The software package ANSYS uses the implicit Newmark's algorithm to solve the transient wave equations.

There are two techniques for the modeling of TOFD using finite element method. The conventional way is using a wedge to generate the incident beam at an expected angle. This method requires much more elements in the model and thus much more computation time and memory. Another method is using ultrasonic beam steering on the specimen surface directly [4,9]. This technique, based on the Huygens Principle of phase construction, is equivalent to the wedge-method. The relation 
between the steering angle $\theta_{s}$ of the ultrasonic beam with the time delay for the adjacent element can be shown as [11]:

$$
\sin \theta_{S}=\frac{c \Delta t}{d}
$$

where $c$ is the wave speed of specimen; $\Delta t$ is the time delay of between the adjacent element; $d$ is the inter spacing of the adjacent element.

Diffractive waves from the crack tip have been experimentally researched by Mei-Ling Jin [12], they used ultrasonic diffraction technique to inspect the depth of a crack from a notch by fatigue test. Chun-Jick Kim et al. [13] compared TOFD technique and radiographic method to evaluate to a mock-up specimen and concluded that the TOFD technique could have a more reliable result. Byung-Sik Yoon et al. [14] calculated the ultrasound beam coverage between two probes for various refracted angles and used phased array ultrasonic TOFD to experimentally detect and sizing the defects. SunHeum Lee et al. [15] developed a deconvolution method to solve the dead-zone problem near the subsurface induced by lateral waves. JoonHyun Lee et al. [16] studied the boundary element method and applied it for surfacebreaking crack evaluation.

However, there is no research work on the FEM simulation on the propagation of ultrasonic waves during TOFD technique. In this paper, the finite element package ABAQUS is used to simulate the wave propagation in the solid media. Here, dynamic explicit method is preferred in the ultrasonic simulation. Here both longitudinal- and shear-wave TOFD are investigated using ultrasonic beam steering. In part 3, longitudinal TOFD for vertical defects with different heights is studied and the predicted results are compared. In part 3, at first the shear-wave TOFD is simulated: the wave patterns are studied by three models; then different-height defects are investigated. This study gives a good display of wave interaction with different sizes of cracks and can help in designing parameter set up of practical TOFD measurements.

\section{Longitudinal-Wave TOFD Simulation}

In this part, longitudinal-wave TOFD have been simulated to inspect the crack inside the material. The specimen material is inconel 600 and the wedge is made up of acrylic. The material parameters are shown in Table 1 .

To get high detectability of the TOFD, the transmission energy into the specimen should be high enough. Here the intensity transmission coefficient [17] of the acrylic-In600 is calculated and shown as Fig. 2.

\subsection{Simulation Setup}

From Fig. 2, in order to fire the longitudinal wave into the specimen, a refracted angle of longitudinal wave is set as $50^{\circ}$ and the full FEM model is shown in Fig. 3. There

Table 1 Material parameters in TOFD simulation

\begin{tabular}{|c|c|c|c|c|c|}
\hline & $\begin{array}{c}\mathrm{c}_{\mathrm{p}} \\
(\mathrm{m} / \mathrm{s})\end{array}$ & $\begin{array}{c}\mathrm{c}_{\mathrm{s}} \\
(\mathrm{m} / \mathrm{s})\end{array}$ & $\begin{array}{c}\text { Density } \rho \\
\left(\mathrm{kg} / \mathrm{m}^{3}\right)\end{array}$ & $\begin{array}{c}\mathrm{E} \\
(\mathrm{MPa})\end{array}$ & $\begin{array}{c}\text { Poisson's } \\
\text { ratio } v\end{array}$ \\
\hline $\begin{array}{c}\text { Inconel } \\
600\end{array}$ & 5870 & 3192 & 8426 & 226487.5 & 0.318 \\
\hline Acrylic & 2077 & 998 & 1190 & 3200 & 0.35 \\
\hline
\end{tabular}

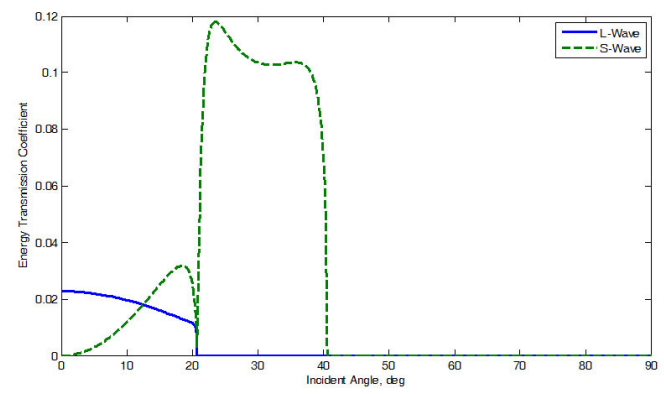

Fig. 2 Schematic diagram of intensity transmission coefficient at acrylic interface as a function of angle: solid line for longitudinal wave; dashed line for shear wave 


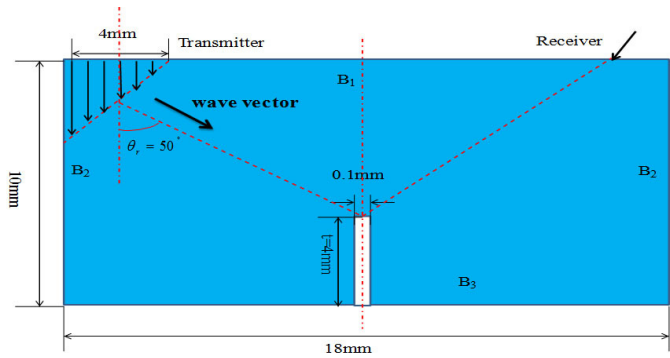

Fig. 3 Geometry model of longitudinal wave TOFD simulation

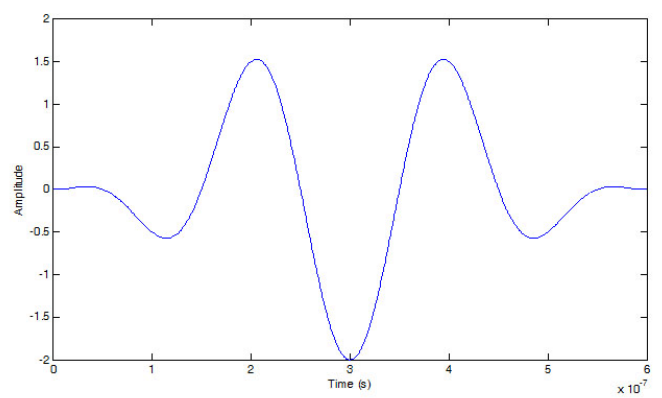

Fig. 4 Three-cycle pulse of $5 \mathrm{MHz}$ centre frequency for excitation of ultrasonic waves

is one point that needs supplementary of the signal reception. Here in this simulation work, the propagated signals are received at a reception point rather than using a finite size of transducer because we want to theoretically discuss the numerical results. In actual situation, the point reception signal can be achieved by application of laser ultrasound transducer.

In this simulation, 4-node 2D-plane-strain elements are used. The element size is chosen as $\Delta x=\lambda / 20=0.06 \mathrm{~mm}$ to get accurate simulation result. The ultrasonic beam is introduced as a transient displacement disturbance on each node with corresponding linear time delay. The time delay for the adjacent element is calculated from Eq. (1) as $\Delta t=7.83 \mathrm{~ns}$. The amplitude of the displacement is $U_{2}=1 \times 10^{-9} \mathrm{~m}$ and the initial pulse form is given by the following equation and shown in Fig. 4.

$$
A(t)=\left\{\begin{array}{cc}
{\left[1-\cos \left(\frac{2 \pi f_{c}}{3} t\right)\right] \cos \left(2 \pi f_{c} t\right),} & 0 \leq t \leq \frac{3.0}{f_{c}} \\
0, & \text { otherwise }
\end{array}\right.
$$

where $f_{c}$ the excitation central frequency, which is $5 \mathrm{MHz}$ in the current FEM model.

To eliminate the wave reflected from left and right boundaries of the specimen, infinite boundary elements are used to simulate the very long specimen. Top and bottom face is set as stress-free boundary. Specimens without notch and with different size $(\mathrm{h}=3,4,5 \mathrm{~mm})$ notches are investigated.

\subsection{Simulation Results and Discussion}

When the disturbance is applied on the surface nodes, the generated waves contain longitudinal wave, shear waves, and surface waves. In order to clearly explain the wave propagation in the TOFD experiment, some snapshots in the specimen has been extracted, shown as Fig. 5.

From Fig. 5, the longitudinal and shear waves are generated inside the specimen; lateral wave and Rayleigh wave are traveling along the surface; lateral wave and shear wave are connected by head wave. The tip diffracted signal is clearly separated for $\mathrm{h}=4 \mathrm{~mm}$. The

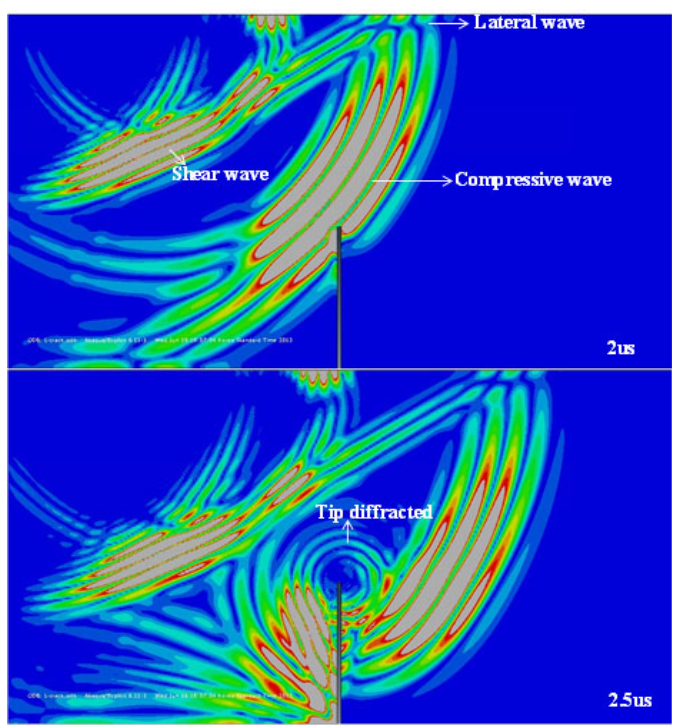

Fig. 5 Snapshots of wave interaction with vertical $\operatorname{defect}(\mathrm{h}=4 \mathrm{~mm})$ at different time steps 
A-scan signal $U_{2}$ at receiving point (shown as Fig. 3) has been extracted, shown as Fig. 6. In the result, lateral wave, tip-diffracted and bottom reflected signals can be seen.

In order to investigate the influence of the notch height, the A-scan signals of different notch heights $\mathrm{h}=3,4,5 \mathrm{~mm}$ are compared, shown as Fig. 7.

From Fig. 7, as expected, the notch height has no influence on the lateral wave. The arriving time of tip diffracted signal decreases while the time for back wall reflected signal increases. The arriving time of tip diffracted signal can be used to size the notch height. The numerical results and theoretical results are compared in Table 2.

From the above simulation results, the longitudinal-wave TOFD is very feasible for the crack inspection and sizing. There are some errors between the theoretical and numerical results, which might be due to the numerical errors. Except these errors, the difference of 3 $\mathrm{mm}, 4 \mathrm{~mm}$ and $5 \mathrm{~mm}$ is well agreed.

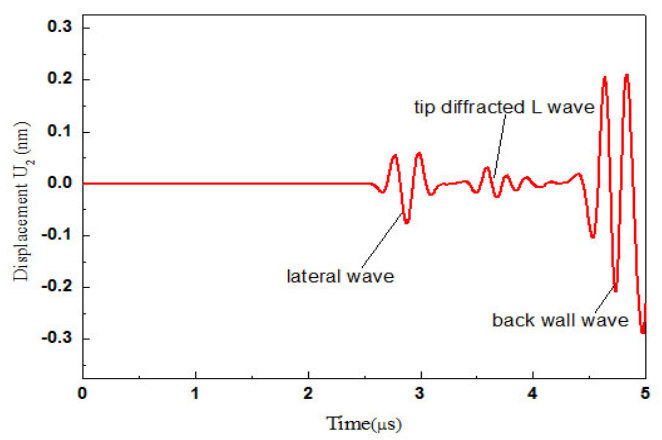

Fig. 6 Simulation A-scan signal with defect $\mathrm{h}=4 \mathrm{~mm}$

Table 2 Time of flight of ultrasonic waves calculated by numerical and theoretical results in L-TOFD simulation

\begin{tabular}{|c|c|c|c|}
\hline Height & Numerical & Theoretical & Error \\
\hline $5 \mathrm{~mm}$ & $3.18 \mu \mathrm{s}$ & $2.97 \mu \mathrm{s}$ & $6.9 \%$ \\
\hline $4 \mathrm{~mm}$ & 3.37 & $3.18 \mu \mathrm{s}$ & $6 \%$ \\
\hline $3 \mathrm{~mm}$ & $3.58 \mu \mathrm{s}$ & $3.41 \mu \mathrm{s}$ & $5 \%$ \\
\hline
\end{tabular}
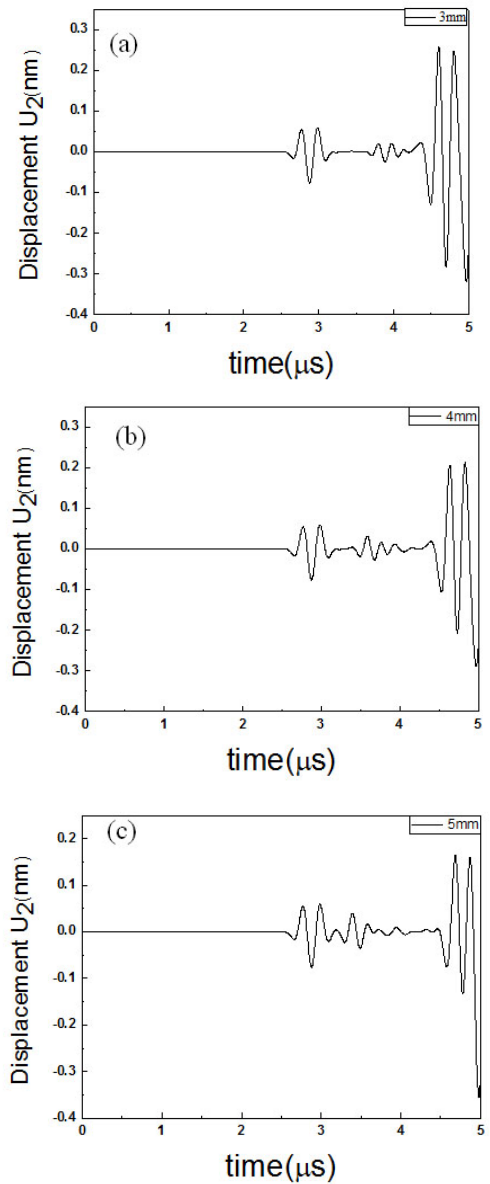

Fig. 7 A-scan signal of different notch heights:

(a) $\mathrm{t}=3 \mathrm{~mm}$; (b) $\mathrm{t}=4 \mathrm{~mm}$; (c) $\mathrm{t}=5 \mathrm{~mm}$

\section{Shear-Wave TOFD Simulation}

As the wavelength of shear wave is almost half of that of longitudinal wave, shear wave shows higher resolution for the crack inspection. Furthermore, the shear wave diffracts much more energy at the obstacles, thus makes shear wave very potential for the TOFD. However, it is difficult to separate the tip diffracted shear wave due to the superposition of many other waves due to the reflection of the top and bottom face, as well as the mode conversion. In order to apply the shear wave for TOFD, different waves should be recognized at first, which can be realized by 
FEM simulation with different boundaries. Here simplified to practical models are built to identify the complicated signals. After that, the difference of A-scan signals between the cracked specimen and perfect specimen are applied for the crack inspection and sizing.

\subsection{Simulation of Different Boundaries}

From Fig. 1, the incident angle is chosen as $\theta_{i}=23.6$ in order to get the highest transmission energy for shear wave. With the Snell's Law, the refracted angle of shear wave is obtained:

$$
\theta_{r}=\sin ^{-1}\left(\frac{c_{s 2}}{c_{p 1}} \sin \theta_{i}\right)=38^{\circ}
$$

The built model for shear-wave TOFD is shown in Fig. 8. The transducer size is set as $2 \mathrm{~mm}$. The element size is set as $\Delta x=\lambda / 20$ $=0.03 \mathrm{~mm}$. The time delay for the adjacent element can be calculated from Eq. (1) as $\Delta t$ $=5.7845 \mathrm{~ns}$.

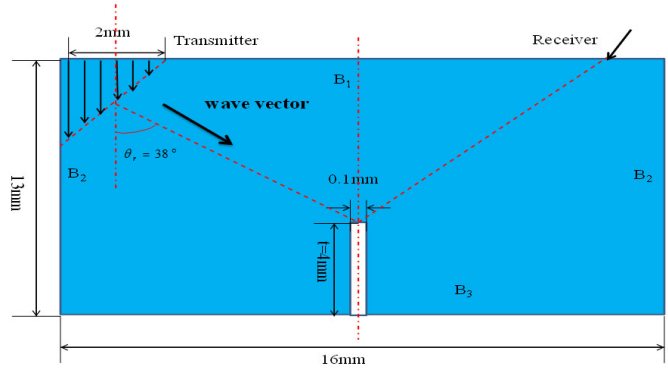

Fig. 8 Model setup for shear-wave TOFD simulation

Table 3 Different boundaries designed for three models

\begin{tabular}{|c|c|c|c|}
\hline & $\mathrm{B}_{1}$ & $\mathrm{~B}_{2}$ & $\mathrm{~B}_{3}$ \\
\hline Model I & Infinite & Infinite & Infinite \\
\hline Model II & Free & Infinite & Infinite \\
\hline Model III & Free & Infinite & Free \\
\hline
\end{tabular}

In order to identify the wave pattern during the shear-wave TOFD, three different models have been built using different boundary conditions, which are expressed in Table 3.

The snapshots of these three models at the same time $(\mathrm{t}=5 \mu \mathrm{s})$ have been captured for comparison. The wave patterns are shown in Fig. 9.

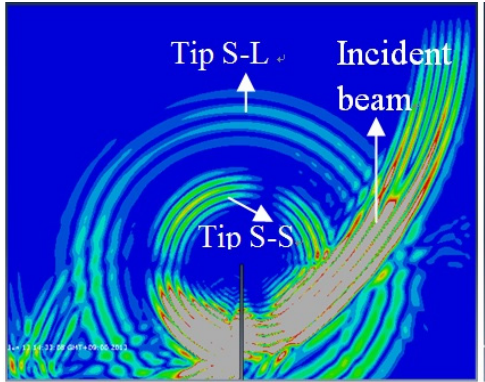

(a)

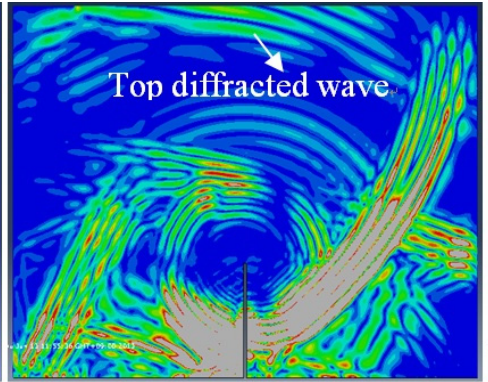

(b)

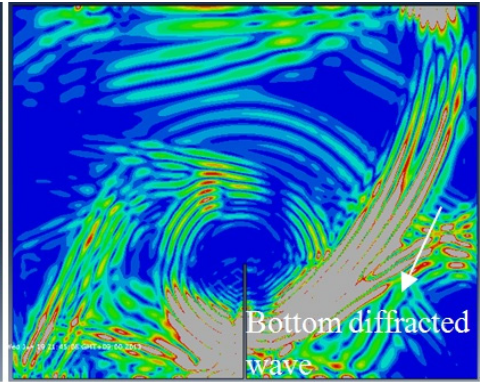

(c)

Fig. 9 Simulation results at $\mathrm{t}=5$ us: (a) Model I; (b) Model II; (c) Model III

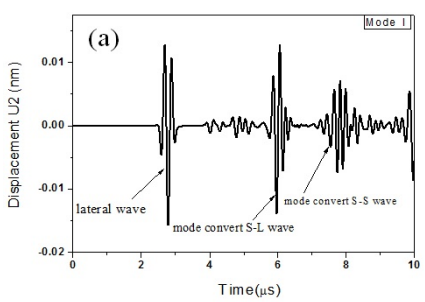

(a)

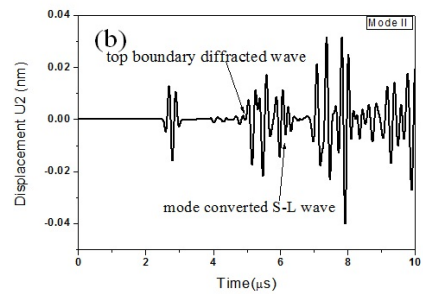

(b)

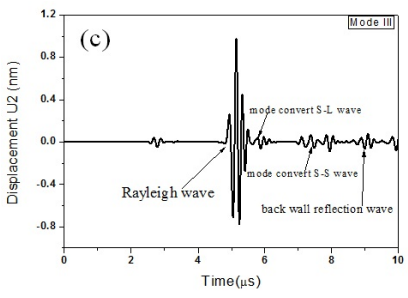

(c)

Fig. 10 A-scan signals comparison: (a) Model I; (b) Model II; (c) Mode III 
From the wave pattern in Fig. 9(a), the tip diffracted shear wave and its converted longitudinal wave are clearly seen. These waves can be identified from the corresponding A-scan signal of Model, which is shown in Fig. 10(a). For the model II, the tip diffracted shear wave and its converted longitudinal wave can also be identified, while a wave reflected from the top interacts with them at the same time. Fig. 9(b) shows the effect of stress-free top boundary. Moreover, the practical case is the Model III with stress-free top and bottom boundaries. From the Fig. 9(c), so many waves are superposed together and it is difficult to extract the tip diffracted signal directly, shown as in the A-scan signal Fig. 10(c).

\subsection{Simulation of Different Crack Heights}

In order to extract the effective diffracted tip shear wave, the bottom and top effects should be excluded. One of the methods is to get the difference time signal between the cracked specimen and the non-cracked specimen. In this way, the top and bottom effects can be get rid of, remaining only the crack-related signal.

Different models, same with Model III, with different crack heights as well as a non-cracked specimen have been built. The A-scan signals of a cracked specimen and a non-cracked specimen are shown in Fig. 11.

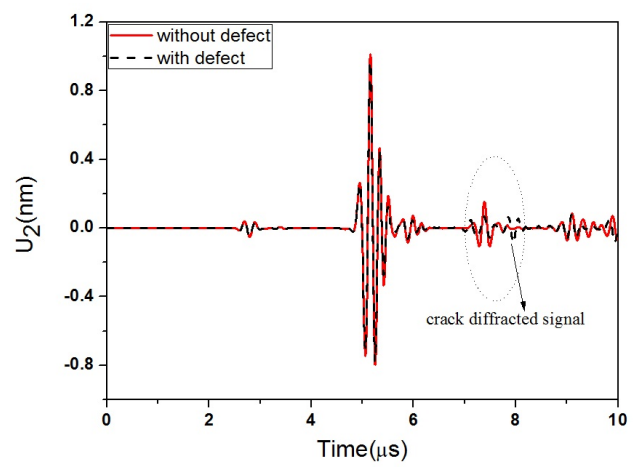

Fig. 11 A-scan signals of defected and non-defected models
From the A-scan signal of defected and nondefected specimen, there is some difference but hard to tell which wave makes such kind of difference. Using the difference of the A-scan signal of defected and non-defected specimen, the crack effect can be magnified and the information can be used for the crack sizing.

The obtained signals difference is shown in Fig. 12(a). From the resultant signal, the tip-diffracted shear wave to longitudinal wave

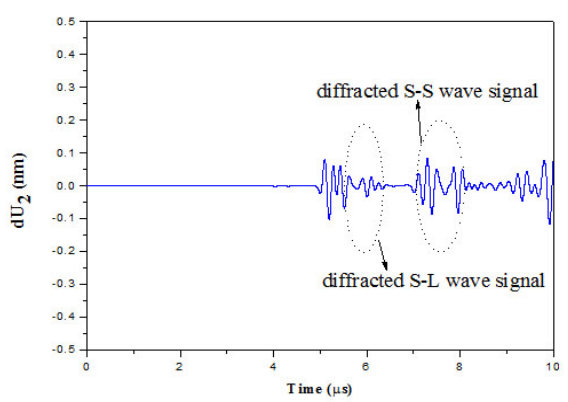

(a)

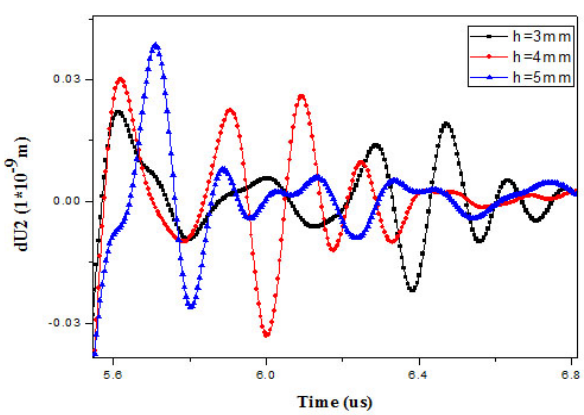

(b)

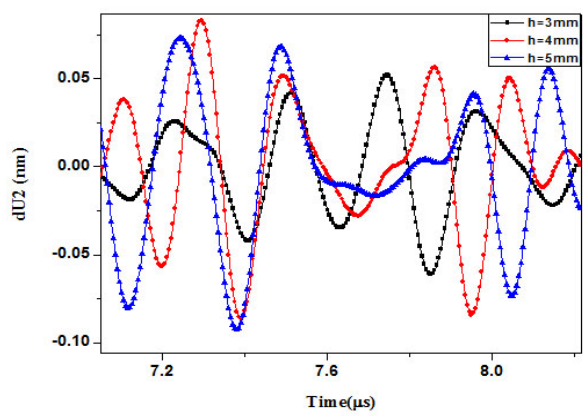

(c)

Fig. 12 Signal difference for different crack heights: (a) A-scan signal difference of $t=4 \mathrm{~mm}$; (b) A-scan signal difference for tip-diffracted $S$ to $L$ wave; (c) A-scan signal difference for tip-diffracted $S$ to $S$ wave 
Table 4 Time of flight of ultrasonic waves calculated by numerical and theoretical results in S-TOFD simulation

\begin{tabular}{|c|c|c|c|}
\hline Height & Numerical & Theoretical & Error \\
\hline $5 \mathrm{~mm}$ & $5.31 \mu \mathrm{s}$ & $5.15 \mu \mathrm{s}$ & $3.2 \%$ \\
\hline $4 \mathrm{~mm}$ & $5.69 \mu \mathrm{s}$ & $5.52 \mu \mathrm{s}$ & $3 \%$ \\
\hline $3 \mathrm{~mm}$ & $6.09 \mu \mathrm{s}$ & $5.91 \mu \mathrm{s}$ & $2.8 \%$ \\
\hline
\end{tabular}

(S-L) is well separated from other waves, while the tip-diffracted shear to shear (S-S) wave is hard to identify, therefore S-L wave can be used for the crack inspection and sizing. The detailed information of different crack heights is shown in Fig. 12(b) and (c).

From Fig. 12(b), the tip-diffracted shear to longitudinal wave signal can be identified and the arriving time decreases with the increasing crack height. The calculated arriving time and its comparison with theoretical results have been summarized in Table 4 .

From the comparison of the arriving time for the tip diffracted shear to longitudinal wave, the result from the theoretical and numerical calculation agree very well. Compared with the error of the longitudinal-wave TOFD, the error is almost two-time smaller, and it is potential to use this method for crack sizing.

\section{Conclusion Remarks}

In this work, finite element method has been applied to study the wave propagation phenomena in TOFD process. Not only the conventional TOFD using tip diffracted longitudinal wave but also the tip diffracted shear wave propagation have been investigated. For the conventional TOFD, the tip signal is well obtained and the crack sizing has been done. The numerical results for the arriving time show a good agreement with the theoretical result.

In order to observe shear wave interaction with the crack, at first three models with different boundaries have been built. The results show that the tip shear signal usually is superposed with other signals from the top and bottom surfaces. Furthermore, to extract the tip shear wave signal, a non-cracked specimen has been introduced as a reference. The A-scan signal difference shows a good detectability for the tip shear to longitudinal (S-L) wave. The arriving time of numerical and geometrical calculations has been compared and these results show two-times smaller error than that of conventional TOFD. The shear-wave TOFD shows a good potential for crack sizing. In spite of this, the shear wave has a slower speed than the longitudinal wave which means that in many cases the signals interaction with the defect will arrive in amongst other spurious signals generated by mode converted compression waves which have travelled further, or by Rayleigh waves. Hence, in many cases, the shear wave signals will be more difficult to interpret than those from compression waves. In case of the anisotropy of material to be inspected, the shear wave may supply a useful way which can be discussed in future work.

\section{Acknowledgement}

This work was supported by the National Research Foundation of Korea (NRF) grant, which is funded by the Korea government (MEST) (No. 2011-0020024).

\section{References}

[1] J. P. Charlesworth and J. A. G. Temple, "Engineering Applications of Ultrasonic Time of Flight Diffraction," Second Ed. Research Studies Press Ltd., pp. 2-10 (2000)

[2] M. G. Silk, "Defect sizing using ultrasonic diffraction," Brit J. NDT, Vol. 21, No. 1, pp. 12-15 (1979)

[3] G. Baskaran, K. Balasubramaniam and C. L. Rao, "Shear wave time-of-flight diffraction 
(S-TOFD) technique," NDT\&E International, Vol. 39, pp. 458-467 (2006)

[4] G. Baskaran, C. L. Rao and K. Balasubramaniam, "Simulation of the TOFD technique using the finite element method," Insight, Vol. 49, No. 11, pp. 641-646 (2007)

[5] G. Baskaran, K. Balasubramaniam, C. V. Krishnamurthy and C. L. Rao, "A ray based model for the ultrasonic time-offlight diffraction simulation of thin walled structure inspection," Journal of Pressure Vessel Technology, Vol. 127, No. 3, pp. 262-268 (2005)

[6] S. Baby, T. Balasubramanian and R. J. Pardikar, "Time-of-flight diffraction(TOFD) technique for accurate sizing of surfacebreaking cracks," Insight, Vol. 45, No. 6, pp. 426-430 (2003)

[7] J. P. Charlesworth and B. M. Hawker, "Inspection of the near-surface defect plate (DDT3) by the ultrasonic time-of-flight technique," Brit J. Non-destruct. Test, Vol. 26, pp. 106-112 (1984)

[8] L. J. Bond, "Methods for the computer modeling of ultrasonic waves in solids," Research techniques in NDT, Vol. 6, pp. 107-150 (1982)

[9] F. Honarvar and S. Khorasani, "Simulation of time-of-flight diffraction (TOFD) technique by finite element method," Online Workshop in www.ndt.net in September (2010)

[10] R. Ludwig and W. Lord, "Finite-element study of ultrasonic wave propagation and scattering in an aluminum block," Materials
Evaluation, Vol. 46, pp. 156-161 (1988)

[11] S. Mondal and T. Satter, "An overview TOFD method and its mathematical model," NDT.net, Vol. 5 No. 4 (2000)

[12] M.-L. Jin,, T.-H. Lee, B.-J. Park and K.-Y. Jhang "Depth sizing of notch fatigue crack using diffracted ultrasonic wave," Journal of the Korean Society for Nondestructive Testing, Vol. 29, No. 5, pp. 405-414 (2009)

[13] C.-J. Kim, J.-G. Jeon and J.-T. Kim, "Comparison of TOFD and radiographic testing for a mock-up specimen," Journal of the Korean Society for Nondestructive Testing, Vol. 28, No. 1, pp. 64-69 (2008)

[14] B.-S. Yoon, Y.-S. Kim, H.-J. Lee and Y.-H. Lee, "A study on TOFD inspection using phased array ultrasonic technique," Journal of the Korean Society for Nondestructive Testing, Vol. 25, No. 4, pp. 304-310 (2005)

[15] S.-H. Lee, S.-H. Kim, Y.-H. Kong and W.-H Lee, "Deconvolution method for TOFD technique," Journal of the Korean Society for Nondestructive Testing, Vol. 19, No. 6, pp. 420-425 (1999)

[16] J.-H. Lee, S.-I. Lee and Y.-H. Cho, "A study on scattered field of ultrasonic wave using the boundary element method," Journal of the Korean Society for Nondestructive Testing, Vol. 20, No. 2, pp. 130-137 (2000)

[17] J. L. Rose and P. B. Nagy, "Ultrasonic waves in solid media. Part 3 Reflection and transmission of ultrasonic waves," $J$. Acoust. Soc. Am. 107, pp. 1-27 (2000) 